\title{
Sharp Reduction of the Secondary Electron Emission Yield from Grooved Surfaces*
}

\author{
M. T. F. Pivi \\ F. K. King, R. E. Kirby, T. O. Raubenheimer, G. Stupakov \\ Stanford Linear Accelerator Center, Stanford University, \\ Stanford, CA 94025 \\ F. Le Pimpec \\ Paul Scherrer Institute 5232 Villigen Switzerland
}

\begin{abstract}
The effect of an artificially-enhanced rough surface on the secondary electron emission yield (SEY) was investigated both theoretically and experimentally. Analytical studies on triangular and rectangular grooved surfaces show the connection between the characteristic parameters of a given geometry to the SEY reduction. The effect of a strong magnetic field is also discussed. SEY of grooved samples have been measured and the results agree with Monte-Carlo simulations.
\end{abstract}

Submitted to Journal of Applied Physics

*Work supported by US Department of Energy contract DE-AC02-76SF00515 


\title{
Sharp Reduction of the Secondary Electron Emission Yield from Grooved Surfaces
}

\author{
M. T. F. Pivi* \\ F. K. King, R. E. Kirby, T. O. Raubenheimer, G. Stupakov \\ Stanford Linear Accelerator Center, \\ 2575 Sand Hill Road, Menlo Park CA-94025, USA \\ F. Le Pimpec \\ Paul Scherrer Institute \\ 5232 Villigen Switzerland
}

\begin{abstract}
The effect of an artificially-enhanced rough surface on the secondary electron emission yield (SEY) was investigated both theoretically and experimentally. Analytical studies on triangular and rectangular grooved surfaces show the connection between the characteristic parameters of a given geometry to the SEY reduction. The effect of a strong magnetic field is also discussed. SEY of grooved samples have been measured and the results agree with Monte-Carlo simulations.
\end{abstract}

Key words: Secondary electron emission, electron-cloud effect, multipacting PACS: 79.20.Hx, 34.80.Bm, 29.27.Bd, 02.70.Uu

\section{Introduction}

In the quest of suppressing multipactor discharge and electron cloud effects (ECE) for future colliders, several methods are under study to lower the secondary emission yield (SEY). Historically, much of the work done to suppress multipacting was developed in the klystron industry $[1,2,3]$. However, the methods used today are in many cases still based on that early work[4]. These include special coatings on metal or ceramic surfaces, surface cleaning,

* Corresponding author

Email address: mpivi@slac.stanford.edu (M. T. F. Pivi). 
beam conditioning and the use of rough or porous surfaces $[5,6,7,8]$. For the positron damping ring (DR) of the International Linear Collider (ILC), it is mandatory for the SEY to be below 1.2, in order to avoid ECE. It is also important to realize that the beam itself and the ECE associated with it, will condition the surface. However, it is imperative to use low SEY materials as a starting point, in order to reduce the in-situ commissioning time. In this paper, we will look, theoretically and experimentally, at the effect and effectiveness of artificially grooving metal surfaces on the SEY.

Based on prediction of reduction of the SEY due to artificially-enhanced surface roughness with triangular grooves [9], an experimental program was started at SLAC for triangular and rectangular grooves [10]. In this paper we consider two types of grooves triangular grooves with angle $\alpha$, and rectangular characterized by the period $b$, dwell width $a$, depth $h$ and a flat top thickness $t$. An initial electron whose trajectory, in Fig.1, is shown in red hits the surface at point $A$ and produces secondary electrons shown with blue lines. Depending on the emission angle, some of the secondary electrons can escape the groove and move away from the surface. Other secondary electrons would hit an inner side of the groove. With some probability they will be absorbed, or they can generate further secondary electrons (which are second generation secondaries) whose trajectories are shown in green. The process may repeat several times until the energy of higher generations becomes too low and they are absorbed by the surface.

a)
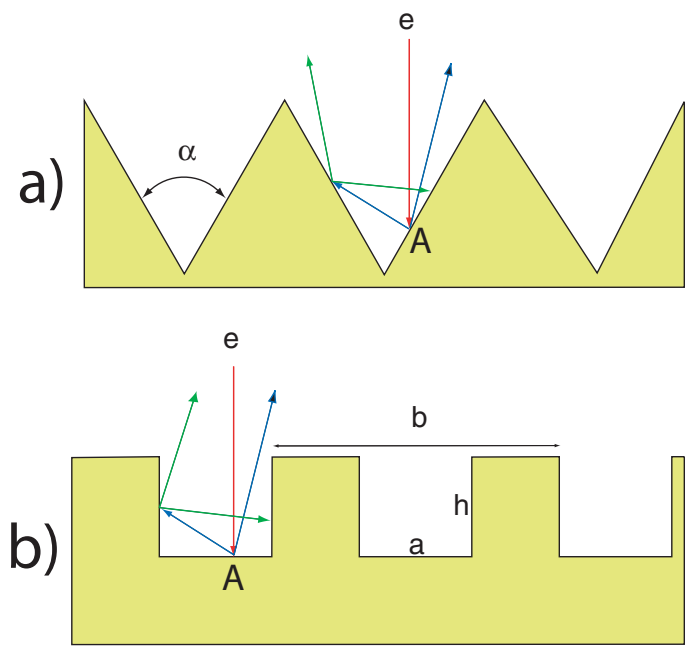

Figure 1. Triangular (a) and rectangular (b) grooves on the surface. Triangular grooves are characterized by the angle $\alpha$. Rectangular grooves have a period $b$, dwell width $a$, depth $h$ and a flat top thickness $t$.

Note that although collisions of secondaries with side walls of the grooves would lead to suppression of the SEY, there is a competing mechanism for triangular grooves that increases the yield. It is due to the fact that a primary electron that is incident perpendicular to the horizontal plane in Fig. 1a hits 
the groove surface at an angle $(\pi-\alpha) / 2$ relative to the sample normal. Since the SEY typically increases toward grazing angle, this means that the number of first generation secondaries will be larger than in the case of normal incidence on a flat surface. This observation shows that it is not obvious whether triangular grooves suppress the effective emission or increase it for a given angle $\alpha$. Simulation results might depend on the specific model of secondary emission. In general, simulation show that a smaller angle results in a lower secondary electron yield.

In our simulation code, we used a subroutine from the POSINST computer code [11] to calculate probability of emission, with a given energy and angular coordinates of the secondary electron. The model implemented in this subroutine is described in Ref. [12]. The angular distribution of secondaries is assumed $\propto \cos \theta$, where $\theta$ is the angle with the normal to the surface, and the incidence-angle dependence of the secondary emission yield $\delta$ is given by relation $\delta \propto\left[1+r_{1}\left(1-\cos ^{r_{2}} \theta_{0}\right)\right]$ where $r_{1}$ and $r_{2}$ are positive numbers that depend on the properties of the surface. In the simulation we assumed that primary electrons hit the surface normal to the averaged plane (as shown in Fig.1). To make calculations faster, we simulated only first 2 or 3 generations of the electrons with about $2 \times 10^{4}$ incident electrons per groove. An effective SEY was obtained by averaging over the groove period. The secondary energy spectrum is a fundamental parameter used in the simulation to take into account the energy distribution of the emitted electrons at the location of the primary incident electron. The emitted energy spectrum of the secondary electrons $\frac{d \delta}{d E}$ can be computed as

$$
\frac{d \delta}{d E}=f_{1, e}+f_{1, r}+\frac{d \delta_{t s}}{d E}
$$

where

$$
\frac{d \delta_{t s}}{d E}=\sum_{n=1}^{\infty} \frac{n P_{n, t s}\left(E_{0}\right)\left(E / \epsilon_{n}\right)^{p_{n}-1} e^{-E / \epsilon_{n}}}{\epsilon_{n} \Gamma\left(p_{n}\right) P\left(n p_{n}, E_{0} / \epsilon_{n}\right)} \times P\left((n-1) p_{n},\left(E_{0}-E\right) / \epsilon_{n}\right)
$$

represents the energy distribution of the so called true secondary electrons to be distinguished from the energy distribution $f_{1, e}$ of the elastically reflected electrons and the energy distribution $f_{1, r}$ of the re-diffused electrons from the bulk of the material [12]. The conventional picture of secondary emission can be summarized as follows: When a steady current of electrons impinges on a surface, a certain portion $I_{e}$ is backscattered elastically while the rest penetrates into the material. Some of these electrons scatter from one or more atoms inside the material and are reflected back out. These are the so-called "re-diffused" electrons, and we call the corresponding current $I_{r}$. The rest of the electrons interact in a more complicated way with the material and yield 
the so-called "true-secondary electrons", whose current we call $I_{t s}$. The yields for each type of electron are combined linearly to give the total SEY of the material.

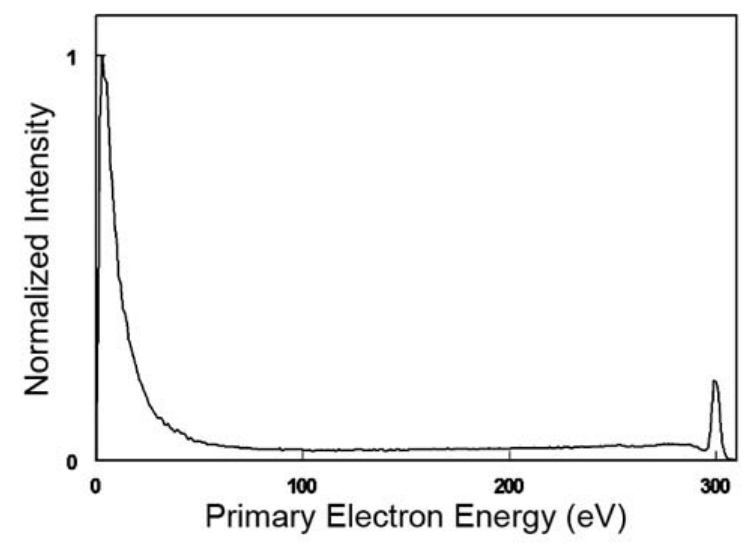

Figure 2. Measured energy distribution of secondary electrons emitted by a TiN surface when impacted by a $300 \mathrm{eV}$ incident electron beam

The distribution of energy of secondary electron emitted from a TiN surface by a $300 \mathrm{eV}$ incident primary electron beam is shown in Fig.2. At $3 \mathrm{keV}$ the distribution of energy of the secondaries is very similar, most of the secondaries having an energy comprise between $0 \mathrm{eV}$ and $50 \mathrm{eV}$.

\section{Grooved Surfaces Simulation Results}

It is interesting to note that the effective SEY does not depend on the size of the grooves and is only a function of the angle $\alpha$. This gives certain flexibility in the practical choice of the dimensions of the grooves. Of course, this independence of SEY on the size of the grooves holds only within some limits and breaks down when the size of the groove becomes comparable to the stopping range of the incident electrons in metal.

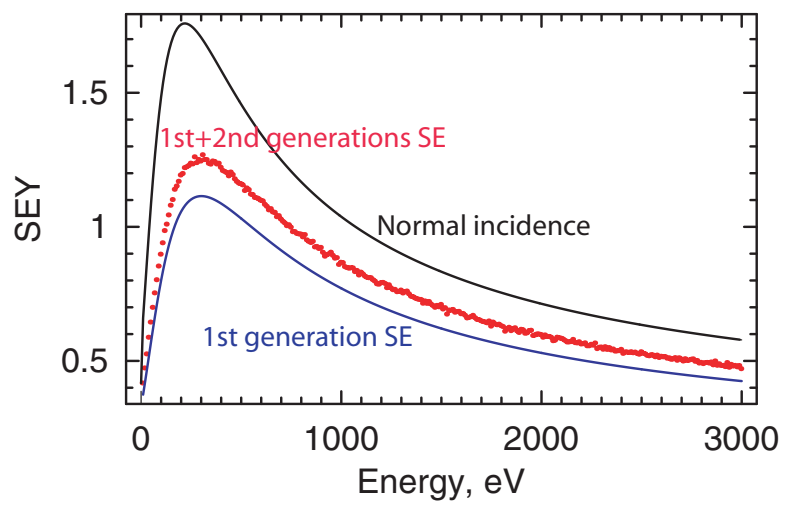

Figure 3. SEY as a function of incident energy for triangular grooves with $\alpha=60^{\circ}$. 
The result of simulations for triangular grooves with angle $\alpha=60^{\circ}$ on a copper surface with maximum SEY $\delta_{\max }=1.75$ is shown in Fig.3. The top curve gives the reference value of $\delta(E)$ (where $E$ is the incident energy of the primary electron) for a flat surface (without grooves) at normal incidence. The blue curve is the effective SEY with grooves when only first generation of secondaries is taken into account (that is each secondary electron is assumed to disappear wheen it hits a wall). The red dots show the result of simulation with two generations of secondaries taken into account (second generation secondary electrons do not produce secondaries when they hit the wall). As is seen in the picture, the maximum effective SEY decreases to a value of about 1.3 in this case.

The average energy computed from the secondary electrons energy distribution of first and second generation as a function of the primary electron energy is shown in Fig.4. With each consecutive generation of secondaries, the average energy goes down and for the second generation, it becomes smaller than the energy corresponding to the maximum of SEY (about $200 \mathrm{eV}$ ). This decrease of the average energy for higher generations of secondary electrons indicates that they should not contribute much into the total effective yield for a grooved surface.

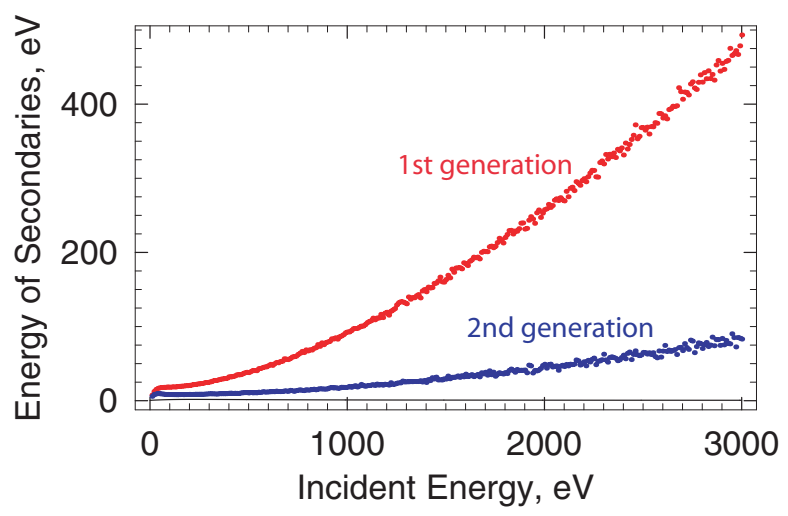

Figure 4. Energy of secondary electrons for triangular grooves with $\alpha=60^{\circ}$.

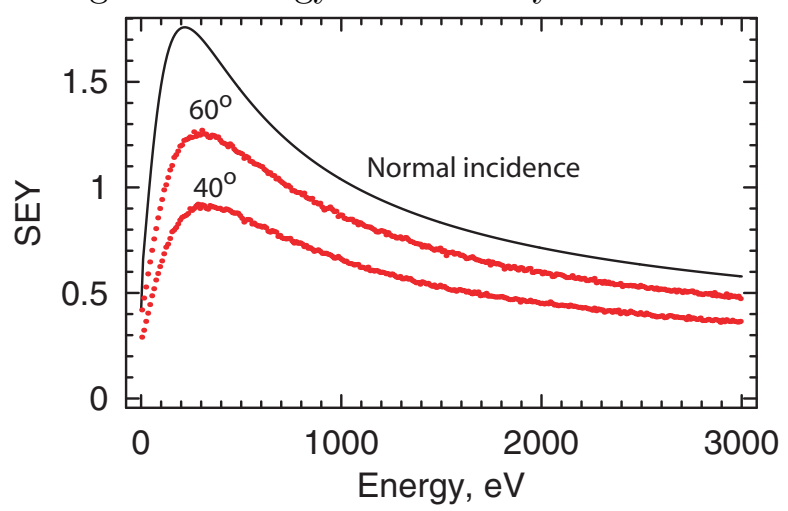

Figure 5. Comparison of the effective SEY for $60^{\circ}$ and $40^{\circ}$ grooves.

In Fig.5, we compare results for grooves with the angle $\alpha$ equal to $40^{\circ}$ and $60^{\circ}$ 
(and the reference case of the flat surface for normal incidence). As one can expect, the smaller angle results in the stronger suppression of the emission, with the maximum value of $\delta$ for $\alpha=40^{\circ}$ approximately equal to $\delta_{\max }=0.9$.

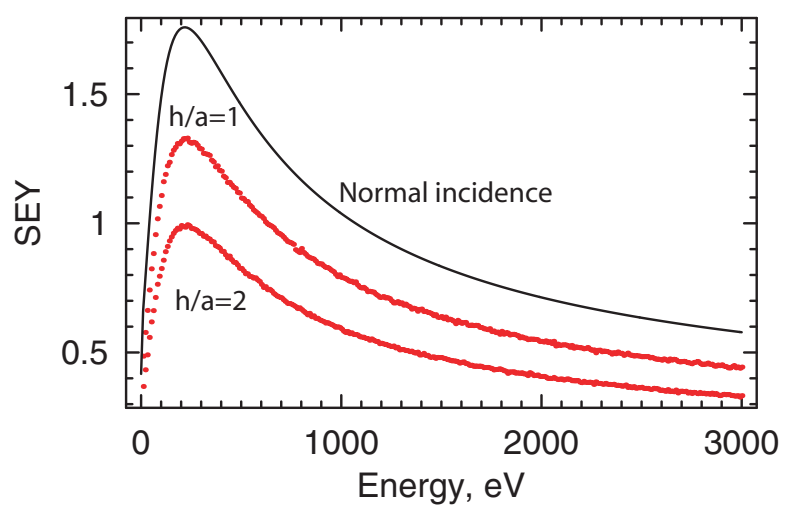

Figure 6. Rectangular grooves with $a=\frac{2}{3} b$.

Fig. 6 shows results of the simulation for rectangular grooves with $a=\frac{2}{3} b$. Two red dotted lines correspond to different aspect ratios of the grooves: the bottom one is for $h / a=2$ and the middle one corresponds to $h / a=1$. The top curve gives the reference value of $\delta(E)$ for a flat surface for normal incidence. As in the case of triangular shapes, deeper grooves show higher suppression of secondary emission.

We also simulate the variation of the secondary emission yield with the rectangular grooves parameters. Fig.7 shows the dependence of the yield with the period-to-depth ratio $h / b$ and for different width-to-thickness $a / t$ values, where $t$ is the flat top groove thickness. For these simulation, we assume In these simulation, we have assumed the reference flat surface without grooves with a peak SEY of 1.75 .

We manufactured a rectangular-grooved sample in copper OFE material and measured the secondary electron yield in the analysis chamber. Sample dimensions are $\mathrm{h}=5 \mathrm{~mm}, \mathrm{~b}=2 \mathrm{~mm}, \mathrm{a}=1.8 \mathrm{~mm}, \mathrm{t}=0.25 \mathrm{~mm}$, and thus with a ratio $a / t=7.2$. On the lower Right of Fig.7, the expected SEY from simulations for a sample with above dimensions is compared to the measurements. Both measurements and simulations of the secondary yield confirm that the secondary electron yield is well below one. The difference between measurements and simulations is within an error of 10-15\%. Fig.7 shows that a good reduction of the SEY for rectangular grooves is obtained with a large depth-to-period ratio $\mathrm{h} / \mathrm{b}$ and a large dwell width-to-thickness ratio a/t. 


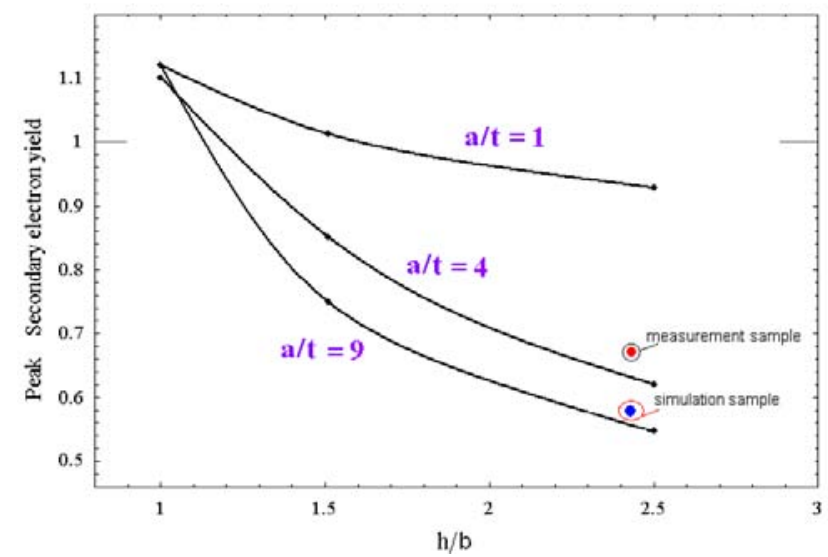

Figure 7. Simulation of secondary yield, of rectangular grooves in magnetic field free region, as a function of $h / b$ ratio for different $a / t$ ratio, where $t$ is the thickness of the flat top groove. On the lower right is also shown the expected SEY of a copper sample compared to effective measurements, with sample dimensions $h=5 \mathrm{~mm}, \mathrm{~b}=2 \mathrm{~mm}$, $\mathrm{a}=1.8 \mathrm{~mm}, \mathrm{t}=0.25 \mathrm{~mm}$ and thus $\mathrm{a} / \mathrm{t}=7.2$. For the simulation, the reference flat top surface has a peak SEY of 1.75 .

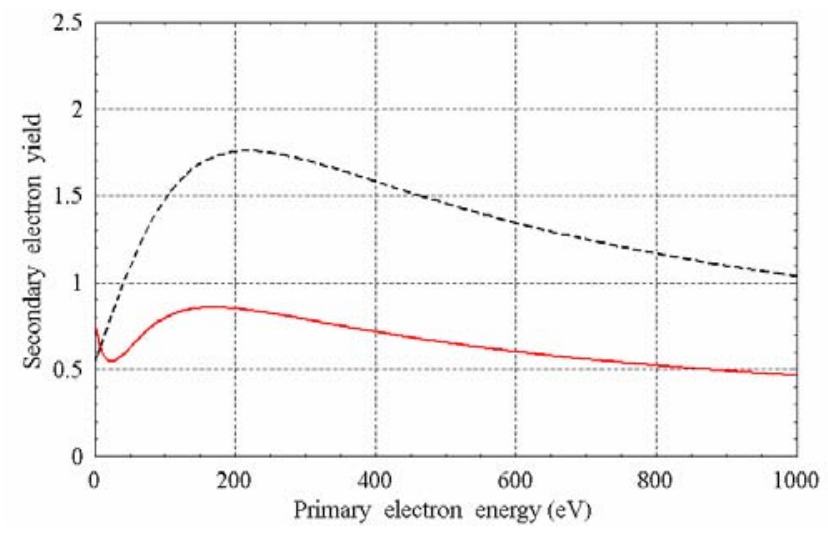

Figure 8. Simulation of rectangular and triangular grooves in $0.2 \mathrm{~T}$ magnetic field. The SEY of a flat surface used as reference in field free region is shown in dashed line. Solid line represents rectangular groove surface with parameters: period $=0.25$ $\mathrm{mm}$ depth $=0.25 \mathrm{~mm}$ width $=0.025 \mathrm{~mm}$.

\section{Effect of Magnetic Field}

Even a weak magnetic field will change the orbits of secondary electrons and affects their collisions with grooved surfaces. For example, for a $200 \mathrm{eV}$ electron, the Larmor radius $r_{L}$ in $1 \mathrm{~T}$ magnetic field is about 25 microns, which is most likely much smaller than the size of the groove. In a dipolar $\mathbf{B}$ field, and for a fully grooved chamber It is easy to understand that in the limit when $r_{L}$ is much smaller than the size of triangular grooves, the effective SEY does depends neither on $r_{L}$ nor the size of the grooves and is only a function of the angle $\alpha$. The reason for this is that in the limit $r_{L} \rightarrow 0$ most of the secondaries in their spiraling motion can collide only with the tilted side surface of the 
groove in the immediate vicinity of the emission point. This remark is not true for electrons having a momentum parallel to $\mathbf{B}$ and produced where the field and the beam chamber are perpendicular. Those electrons can be extracted from the grooves and travel across the chamber to hit the opposite wall, and produced secondaries. However, the emitted electrons will then be driven to the bottom of the grooves, thence disappearing.

We have simulated two different cases of grooves bathed in a $0.2 \mathrm{~T}$ magnetic field. The field applied in the simulation is perpendicular to the substrate of the groove sample. The Larmor radius in such magnetic field is about 125 microns for a $200 \mathrm{eV}$ electron. This is the typical magnetic field of dipolar bends for the ILC positron damping ring. The results of the simulations for a rectangular groove sample are shown in Fig.8; the peak secondary yield can be lowered below 1. The results for triangular grooves of angles $\alpha=60^{\circ}$ and $\alpha=40^{\circ}$ are shown in Fig.9 and 10 respectively. The solid black curve in these plots are reference curves of a flat surface impinged at normal incidence with a $\delta_{\max }=1.75$. Three color dotted curves show the effective SEY with 1, 2, or 3 generations of secondaries taken into account. Surprisingly, we found that with magnetic field, the contribution of higher generations of secondaries becomes more important than for the case without magnetic field. Our result shows that for 60 degree grooves, with 3 generation of secondaries, the total SEY actually exceeds the emission of a flat surface for energies above $\approx 300 \mathrm{eV}$. However, a smaller angle, 40 degrees, shows a noticeable suppression in the energy range below $700 \mathrm{eV}$.

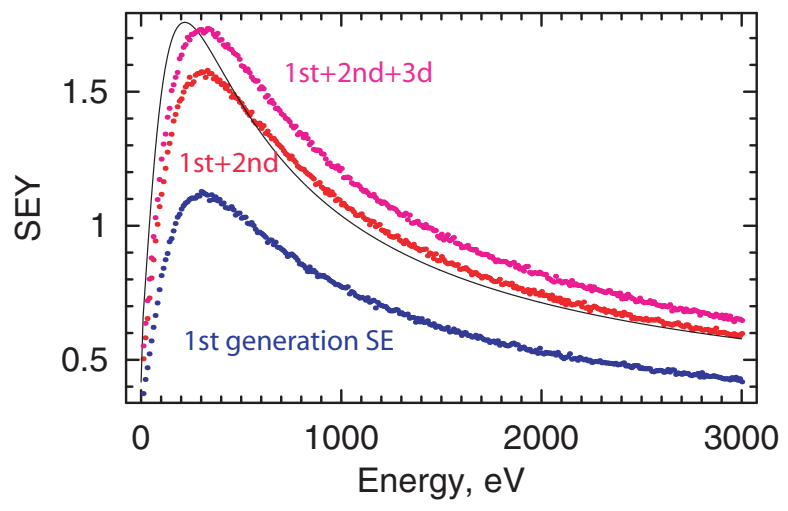

Figure 9. Secondary emission yield in a $0.2 \mathrm{~T}$ magnetic field, $\alpha=60^{\circ}$.

Simulations show that the trend of SEY reduction continues for smaller angles. A triangular groove angle of 20 degrees is effective to decrease the SEY well below unity. For a more complete analysis of triangular groove with smaller angles see $[13,14]$.

The use of sawtooth has been investigated to minimize the photodesorption outgassing of the chamber wall [15]. This idea was re-used, in the case of the LHC (large Hadron Collider), to diminish the production of photoelectron from the copper beam screen, those photoelectrons being the main cause of 


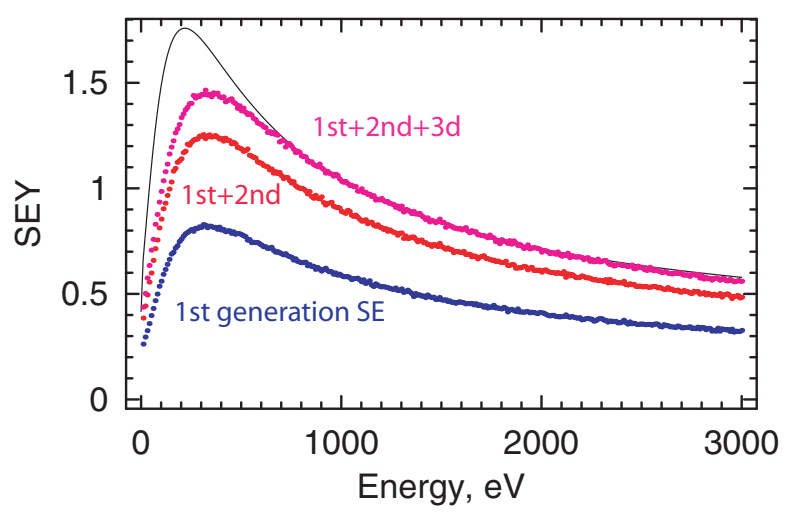

Figure 10. Secondary emission yield in a $0.2 \mathrm{~T}$ magnetic field, $\alpha=40^{\circ}$.

the ECE $[16,17]$. A sawtooth design is also a possible remedy for the ECE in the ILC damping ring, where magnetic fields are present.

\section{Experimental Studies}

\subsection{Experimental setup}

The system used to measure the SEY is described in detail in [18]. Measuring techniques included x-ray photoelectron spectroscopy (XPS) and residual gas analysis (RGA).

The SEY $(\delta)$ definition is determined from the following equation

$$
\begin{aligned}
& \delta=\frac{\text { Number of electrons leaving the surface }}{\text { Number of incident electrons }} \\
& \delta=1-\frac{I_{T}}{I_{P}}
\end{aligned}
$$

In practice equation (4) is used because it contains parameters directly measured in the retarding target potential experiment. $\mathrm{I}_{P}$ is the primary current (the current leaving the electron gun and impinging on the surface of the sample) and $I_{T}$ is the total current measured on the sample $\left(I_{T}=I_{P}-I_{S E}\right)$. $\mathrm{I}_{S E}$ is the secondary electron current leaving the target.

The SEY is measured, at normal incidence, by using a gun capable of delivering a scanning electron beam of $0-3 \mathrm{keV}$, working at a set current of $2 \mathrm{nA}$ and having a $0.4 \mathrm{~mm}^{2}$ spot size on the target. To scan grooved surfaces the gun can be set into a raster mode. In this mode the primary electron beam is rapidly deflected, like in a cathodic tube, and scan an area of $\mathrm{a} \mathrm{cm}^{2}$. For each given 
energy an average current over 100 measurement is returned to the computer by the electrometer. The variation between SEY measured on a flat surface in the raster mode vs the flat mode is less than $2 \%$, measurement obtained on a fully flat reference sample.

The measurement of the SEY is done while biasing the sample to $-20 \mathrm{~V}$. This retarding field repels most secondaries from adjacent parts of the system that are excited by the elastically reflected primary beam. The primary beam current as a function of the primary beam energy is measured and recorded each time before an SEY measurement, by biasing the target to $+150 \mathrm{~V}$, and with the same step in energy for the electron beam. A fresh current lookup table is created with each measurement. The SEY measurement, over the 0$3 \mathrm{keV}$ range, takes around 5 minutes. The first point of the data is taken at $10 \mathrm{eV}$. At $0 \mathrm{eV}$, hence no primary electron beam, the $\mathrm{SEY}$ is artificially set to 1.

\subsection{Experimental results}

In order to obtain a variety of triangular groove profiles, the samples were machined by electrical discharge (EDM). All samples have a diameter of $2.54 \mathrm{~cm}$. EDM creates a thick oxide of $\mathrm{Al}_{2} \mathrm{O}_{3}$ on the $\mathrm{Al} 6063$ alloy, explaining the high value of the SEY max for the flat surface. All the SEY measurements presented in this paper were made in a magnetic free region. All the grooves measurement were obtained in a raster mode. Measurement on the flat part of a half grooved half flat sample were obtained on point mode.

The first experimental confirmation of the sharp reduction predicted by the simulation were obtained on triangular grooved sample made of $\mathrm{Al} 6063$, and then coated with $130 \mathrm{~nm}$ of TiN Fig.11. Another example of SEY reduction from a grooved $\mathrm{Al}$ sample is shown in Fig.12. The grooves of this sample $(0.2 \mathrm{~mm})$ are not as deep as for the other sample shown in Fig.11, with the angle $\alpha \sim 40^{\circ}$. A similar reduction of the SEY is seen. 

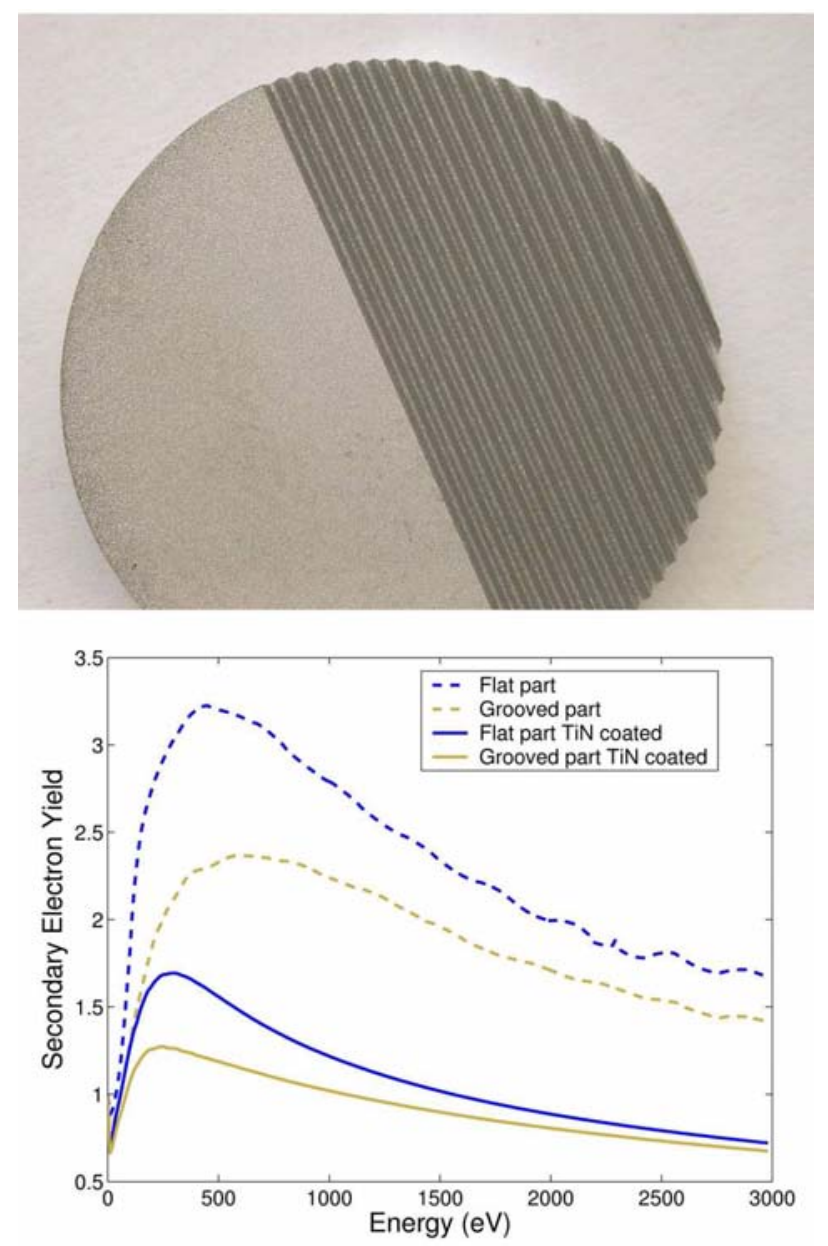

Figure 11. Al 6063 alloy sample half flat and half grooved, 1 inch diameter. Triangular grooves are $1 \mathrm{~mm}$ deep and full opening angle, $\alpha$, is $40^{\circ}$ (Top). SEY results obtained, at normal primary incidence, before and after TiN coating (Bottom).

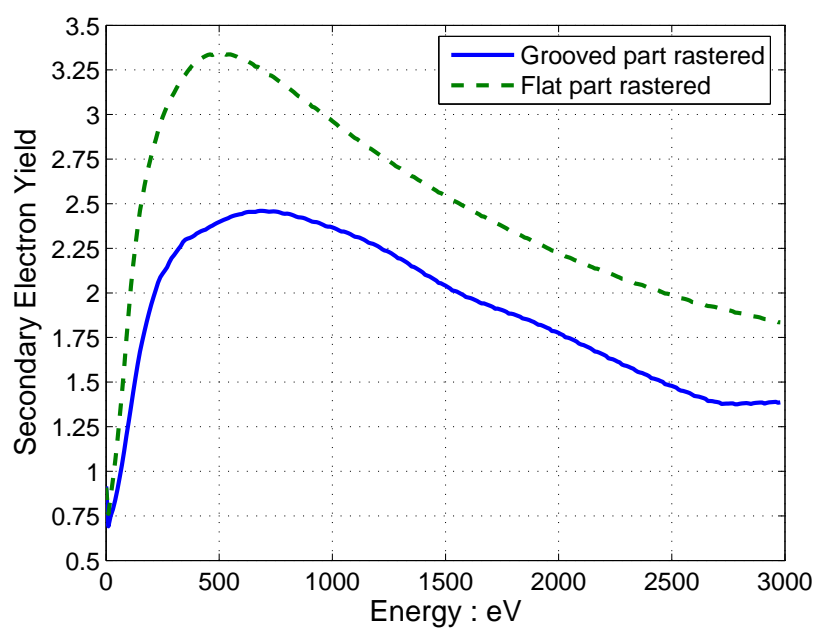

Figure 12. EDM Al sample $\# 7$ triangular grooves of $\alpha \sim 40^{\circ}$, and a depth $=0.2 \mathrm{~mm}$. Rectangular-grooved surfaces were tested, with examples for $\mathrm{Cu}$ shown in 
Fig.13. The fully grooved rectangular sample have grooves of a height $\mathrm{h}=5 \mathrm{~mm}$, a distance between the right and left side of the grooves wall of $\mathrm{a}=1.8 \mathrm{~mm}$, and a fin thickness of $t=0.254 \mathrm{~mm}$. The slanted wall are inclined with a 20 degrees angles and grooves dimensions are $\mathrm{h}=1.0 \mathrm{~mm}, \mathrm{a}=0.35 \mathrm{~mm}, \mathrm{t}=$ $0.05 \mathrm{~mm}$.

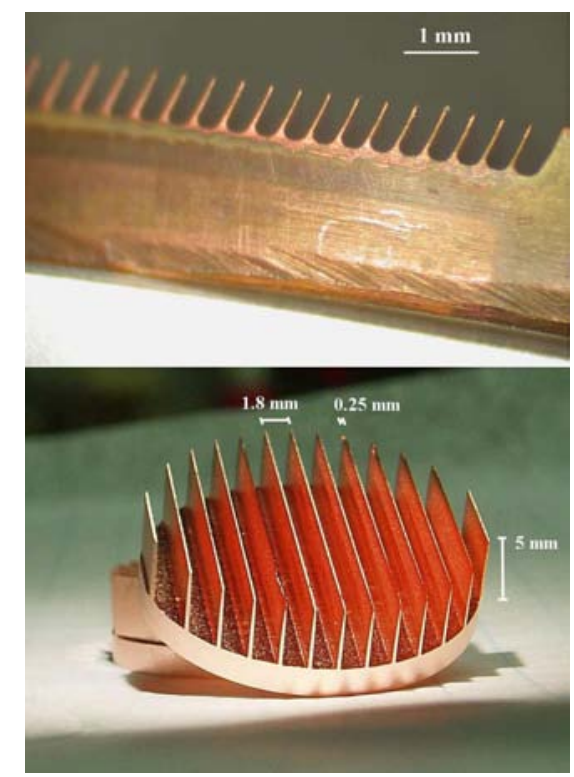

Figure 13. Grooved $\mathrm{Cu}$ samples. On the top figure the half flat and half slanted groove sample and on the bottom figure a fully rectangular $\mathrm{Cu}$ sample $(\mathrm{h} \times \mathrm{a} \times \mathrm{t}$ $=5 \times 1.8 \times 0.254[\mathrm{~mm}])$.

SEY results obtained from the grooved copper samples are presented in Fig.14 and Fig.15. In Fig.14, the SEY reduction from a flat $\mathrm{Cu}$ surface to a slanted grooved one is almost $50 \%$ (solid lines). The effect of the rectangular grooves brings a reduction up to $60 \%$ from a flat $\mathrm{Cu}$ sample (dashed line). The simulation shows that for a ratio $\mathrm{h} / \mathrm{a}=2$ the reduction is close to $40 \%$, Fig. 6 . The $\mathrm{h} /$ a ratio for our sample is $\sim 2.8$, hence bringing more reduction, as expected from the simulations.

In Fig.15, the angle effect from the impinging primary beam was investigated. As for flat surfaces, the more grazing the beam is, the higher the SEY becomes; dashed lines comparison. As the primary electron beam does not scan the full sample as a square, it is important to see the effect of a different orientation of the impinging beam on the SEY. The sample was then rotated $90^{\circ}$ about surface normal its previous position and re-measured (solid lines). In this configuration, the impinging beam scan the inside of the valley a ridge and then the other valley. The SEY results are similar to the one obtained when the electron beam is focused on one point down in the valley. Finally, inversely to the energy of the beam, the surface scanned becomes smaller, the design of the electron gun with fixed deflecting plate potentials being the reason. 


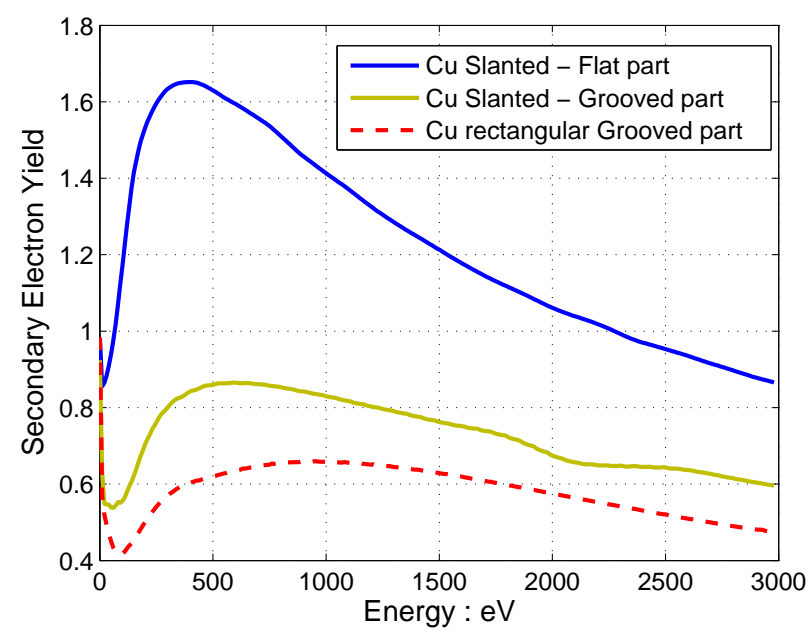

Figure 14. SEY reduction on the half slanted sample groove side compare to its flat part (solid line), see sample in Fig.13 top figure. SEY reduction due to rectangular grooves of the sample shown in Fig.13 bottom figure, (dashed line).

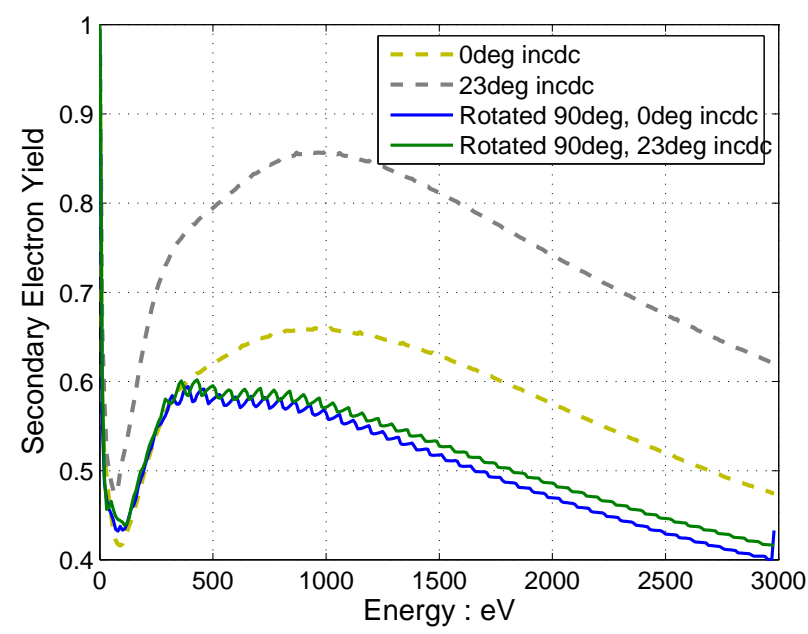

Figure 15. Variation of the SEY on the rectangular grooved $\mathrm{Cu}$ sample (Fig.13 bottom figure) at $0^{\circ}$ and $23^{\circ}$ electron beam incidence angle respect to the normal of the surface, and in function of the grooves orientation relative to the incoming electron beam.

Because the results obtained with $\mathrm{Cu}$ samples were very encouraging, we prepared rectangular-grooved Al sample coated with TiN thin film and NEG coating (TiZrV), Fig.16. The SEY max from a clean technical $\mathrm{Cu}$ sample is between 1.6 to 2.2. Technical Al SEY max is above 2.3. Hence, Al chamber must be coated for the ILC damping rings. A triangular grooved coated $\mathrm{Al}$ sample would be at the permissible SEY limit set for ECE in the ILC DR field free regions, Fig.11.

Results for the $\mathrm{Al}$ coated rectangular grooved sample are presented in Fig.17 and Fig.18. The SEY max is reduced below 1, as it was the case for the $\mathrm{Cu}$ samples. Moreover, in the case of the NEG coated sample, activation reduces 
the SEY further. Upon activation TiZrV NEG, deposited on a flat surface, shows the SEY max dropping from $\sim 1.8$ to around 1.2 [8]. In the case of the non activated NEG sample, $\mathrm{h} / \mathrm{a}=5, \mathrm{~h} / \mathrm{b}=1.67$ and $\mathrm{a} / \mathrm{t}=1$. The value of the SEY obtained fits well with the simulation prediction for the given parameters, Fig.7.

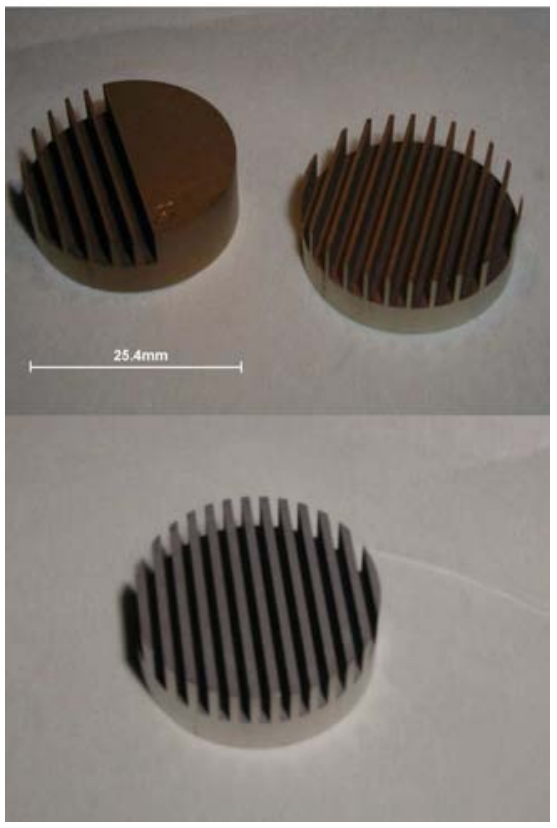

Figure 16. TiN/Al sample $(\mathrm{h} \times \mathrm{a} \times \mathrm{t}=5 \times 2.5 \times 0.6[\mathrm{~mm}])$ Top picture, and TiZrV $/ \mathrm{Al}(\mathrm{h} \times \mathrm{a} \times \mathrm{t}=5 \times 1 \times 1[\mathrm{~mm}])$ Bottom picture. Samples were coated at the L. Berkeley Natl. Laboratory LBNL.

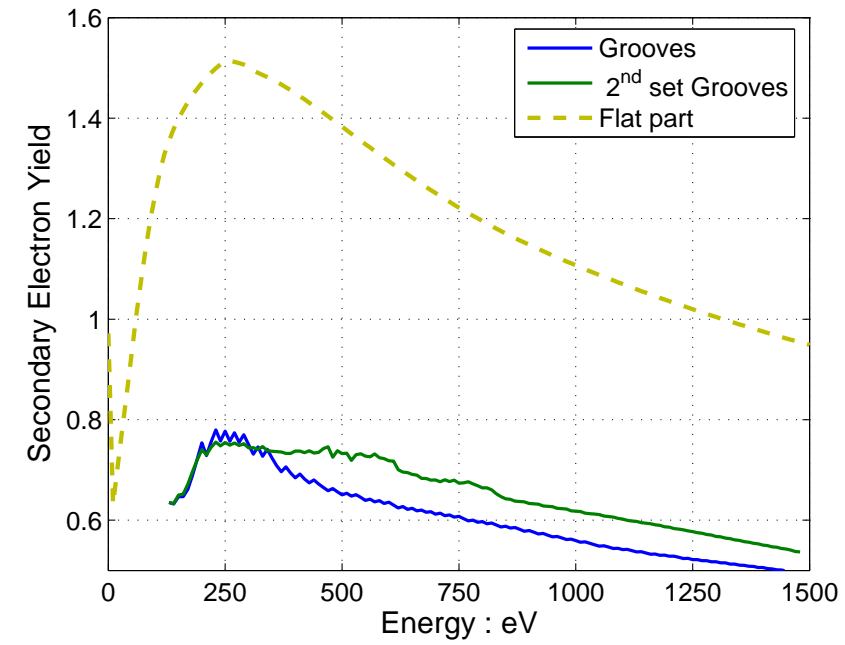

Figure 17. SEY reduction of a grooved TiN coated Al sample, see sample in Fig.16 top figure. Comparison between the rectangular grooves, rastered, and the flat part. 


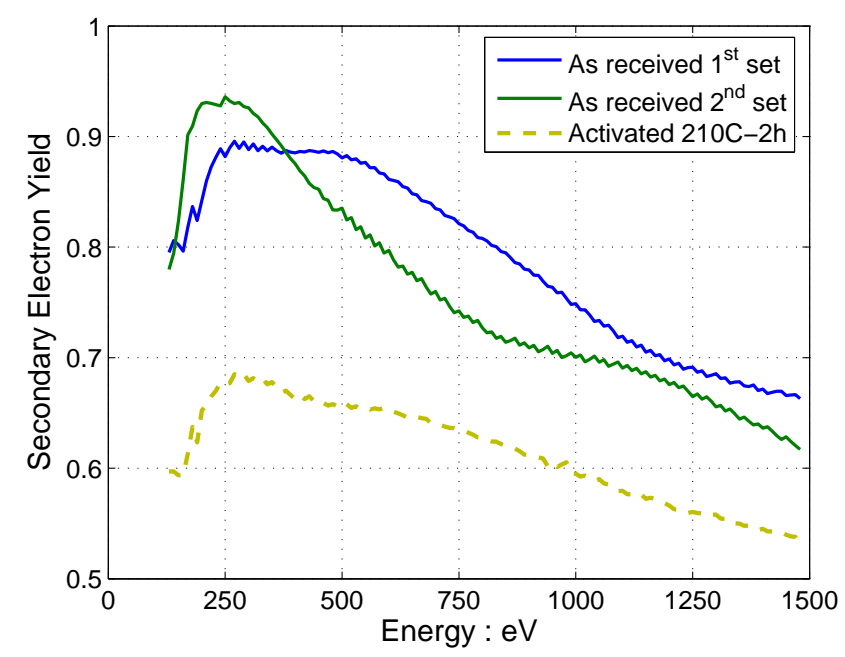

Figure 18. SEY reduction of a grooved TiZrV getter coated Al sample, see sample Fig.16 bottom figure. SEY comparison between non activated and activated NEG.

\section{Summary and Discussion}

We presented results of computer simulations showing that one can suppress SEY using grooves on the surface of the vacuum chamber. The magnitude of suppression depends on the angle of triangular grooves and the aspect ratio of rectangular grooves. The measurements were performed in a magnetic fieldfree region. Simulation and experiments are in good agreement.

Without magnetic field, the suppression depends only on dimensionless parameters that characterize the geometry of the grooves. In the case of triangular shapes, this parameter is the angle $\alpha$. For rectangular grooves there are two such parameters: the aspect ratio $h / a$ and the fraction $a / t$ of the surface occupied by grooves. In case of magnetic field, in the limit $r_{L} \rightarrow 0$, the suppression for triangular grooves also depends only on the angle $\alpha$. Triangular grooves are to be preferred in magnetic field region, since rectangular grooves are effective only when the separation between grooves is comparable to the electron's gyration diameter $a \lesssim 2 \cdot r_{L}$, which might be a technical challenge in the case of strong magnetic fields.

For the cases considered in this paper, we found that SEY suppressions in magnetic field requires triangular grooves with small angles. We address the reader to the references $[13,14]$ for more studies on triangular grooves.

Different samples with different grooves geometries have been measured. All samples did show an improvement when comparing to a flat sample. However, the machining, or extrusion of chamber made with the best geometries measured might not be feasible at a reasonable cost. Some of the first Al grooved samples made with very thin ridges, and narrow canyon, showed damages on 
the ridges, which have occurred during the machining. Application of thin coating, with lower SEY than the bare technical metal, on top of the grooves have helped further reducing the SEY. The homogeneity of the coating along the profile of the groove is not an easy task, since the top of the grooves tend to be coated more than the side walls and the base. The two combined methods (grooving and coating) can be a solution in producing a high-performance chamber.

Introduction of grooves on the surface will change the properties of interaction of the beam with the wall. To minimize the resistive wall impedance, grooves should be oriented along the beam orbit. Another consequence of the grooves is that the beam electric field will be concentrated on the edges of the grooves with a relatively small magnitude of the beam electric field penetrating to the bottom of the grooves. Due to this shielding, secondary electrons emitted near the bottom of the groove will feel a suppressed electric field of the beam. However, the presence of the electric field from the positron beam could trigger the emission of electrons due to field emission from those edges [19]. Nevertheless, field emission will occur for total electric field $(\beta \mathrm{E})$ in excess of a few $\mathrm{GV} / \mathrm{m}$ and it would be unlikely to occur in machines as PEP-II or the ILC DR [20]. A last effect of the presence of the grooves on the chamber is that image current from the beam will run on the ridges of the grooves. Calculation on the ridges temperature rise must be effectuated to ensure that the ridges will not deform and bend, closing up the gap of the grooves and spoiling the effect of the grooves.

\section{Acknowledgement}

The authors are thankful to S. Heifets for useful discussion. We gratefully thanks D. Lee and A. Wolski at LBNL for providing the samples. This work is supported by the US DOE under contracts DE-AC03-76SF00515 and DEAC03-76SF00098

\section{References}

[1] R. A. Kishek, Y. Y. Lau, L. K. Ang, A. Valfells and R. M. Gilgenbach, Physics of Plasmas, 5(5):2120, 1998.

[2] E.W. Hoyt, W.P. Schulz, SLAC-TN-75-003 (1975).

[3] E.L. Garwin, F.K. King, R.E. Kirby and O. Aita, J. Appl. Phys., 61 (1987), p. 1145 .

[4] A. J. Hatch. Nucl. Instrum. Meth., 41(2):261, 1966. 
[5] J.W. Noé. Nucl. Instrum. Meth., A 328:291, 1993.

[6] V. Baglin, J. Bojko, O. Gröbner, B. Henrist, N. Hilleret , C. Scheuerlein and M. Taborelli, The Secondary Electron Yield of Technical Materials and its Variation with Surface Treatments. in EPAC Conf. Proceedings, Vienna, Austria, 2000.

[7] L. Galán, et al., Surface Treatment and Coating for the Reduction of Multipactor and Passive Intermodulation (PIM) Effects in RF Components. In $4^{\text {th }}$ International Workshop on Multipactor, Corona and PIM in Space Hardware, 2003.

[8] F. Le Pimpec, F. King, R.E. Kirby and M. Pivi, Nucl. Instrum. Meth., A 564:44-50, 2006.

[9] A.A. Krasnov. Vacuum, 73:195, 2004.

[10] G. Stupakov and M. Pivi, SLAC-TN-04-045, LCC-0145, 2004.

[11] M. Furman and G. Lambertson. Note LBNL-41123.

[12] M. A. Furman and M. Pivi. Phys. Rev. ST Accel. Beams 5, 124404, 2002.

[13] L. Wang, T. Raubenheimer and G. Stupakov, Suppression of Secondary Emission in a magnetic Field using a sawtooth Surface. in EPAC Conf. Proceedings, Scotland, 2006.

[14] M. Venturini, M. Furman, J-L. Vay and M. Pivi, Modelling of E-cloud Buildup in Grooved Vacuum Chambers Using POSINST. in PAC Conf. Proceedings, New Mexico, USA, 2007.

[15] C.L. Foerster, H.J. Halama and G. Korn, J. Vac. Sci. Technol., A 10(4):2077, 1992.

[16] I.R Collins V. Baglin and O. Gröbner, Photoelectron yield and photoelecytron reflectivity from candidate $\mathrm{LHC}$ vacuum chamber materials with implications to the vacuum chamber design. in EPAC Conf. Proceedings, Stockholm, 1998.

[17] N. Mahne, V. Baglin, I.R. Collins, A. Giglia, L. Pasquali, M. Pedio, S. Nannarone and R. Cimino, Appl. Surf. Sci., 235:221-226, 2004.

[18] F. Le Pimpec, F. King, R.E. Kirby and M. Pivi. J. Vac. Sci. Technol., A (23):1610, 2005.

[19] B.B. Levchenko. On Field Emission in High Energy Colliders Initiated by a Relativistic Positively Charged Bunch of Particles. Technical report, 2006. arxiv:physics/0608135.

[20] B.B. Levchenko. Private communication, May 2007. 